\title{
Resistência à compressão axial do concreto com diferentes direções de carregamento e condições de contorno
}

\section{Concrete axial compressive strength with different loading directions and boundary conditions}

\author{
Matthews Vargas Vaucher Bandeira ${ }^{1}$, Raquel Caroline Zydeck ${ }^{1}$, \\ Luis Eduardo Kosteski ${ }^{1}$, Ederli Marangon ${ }^{1}$
}

\footnotetext{
${ }^{1}$ Programa de Pós-Graduação em Engenharia, Universidade Federal do Pampa, Campus Alegrete, RS, Brasil. e-mail: matthews_vaucher@hotmail.com,raquel.zydeck@gmail.com, luiskosteski@gmail.com, ederlimarangon@gmail.com
}

\section{RESUMO}

Neste trabalho foi analisado experimentalmente a resistência à compressão axial, em corpos de prova cúbicos com $10 \mathrm{~cm}$ de lado, de três traços de concreto com resistências característica das classes de $30 \mathrm{MPa}, 50 \mathrm{MPa}$ e $65 \mathrm{MPa}$. No momento dos ensaios, os corpos de prova cúbicos foram carregados na mesma direção da moldagem, carregamento na direção do lançamento, ou foram girados $90^{\circ}$ para caracterizar o carregamento normal à direção do lançamento. Assim, a direção de aplicação da carga de compressão para a análise da resistência à compressão foi realizada na direção paralela e perpendicular ao lançamento do concreto nos moldes. Também, foi analisado o modo de fratura das amostras quando modificadas as condições de contorno na execução dos ensaios de compressão axial. Na primeira condição de contorno foi usado graxa entre os corpos de prova e os pratos da máquina de ensaios para minimizar o atrito entre eles. Na segunda configuração, placas de aço de $3 \mathrm{~mm}$ de espessura foram coladas nas faces dos corpos de prova maximizando assim o atrito. Dentre as principais conclusões, percebe-se a variação da resistência de acordo com a direção do carregamento. Para o concreto de $50 \mathrm{MPa}$, quando aplicado o carregamento paralelo ao lançamento do concreto, as resistências à compressão foram, em média, 16\% superiores comparado ao carregamento aplicado perpendicular ao lançamento. Em relação às condições de contorno, verifica-se que, quando os corpos de prova estão com suas faces restringidas, há um acréscimo significativo de resistência à compressão devido ao confinamento, independentemente da classe de concreto analisado. É possível verificar uma potencial contribuição ao desenvolvimento de projetos de concreto armado e análise estrutural desse material no ramo da construção civil.

Palavras-chave: resistência à compressão axial; zonas de transição; anisotropia; restrição de apoio; concreto.

\begin{abstract}
In this work the axial compression strength of $10 \mathrm{~cm}$ side cubic specimens of three concrete with characteristic strengths of $30 \mathrm{MPa}, 50 \mathrm{MPa}$, and $65 \mathrm{MPa}$ were analyzed experimentally. The compression load was applied in the same direction and perpendicular to the casting direction of the cubic specimens. The fracture mode was analyzed when the boundary conditions of the tests were modified. In the first boundary condition, grease was applied between the specimens and the machine's plate to minimize friction. In the second boundary condition, $3 \mathrm{~mm}$ steel plates were glued to the sides of the specimens to maximize friction. One of the main results was that the strength change with the load direction. For the $50 \mathrm{MPa}$ concrete, the compression strength was $16 \%$ higher when it was parallel to the casting direction than when it was perpendicular. With respect to the boundary conditions, it is found that when the specimen sides were restricted, there was an increase in the compression strength, independently of the concrete class. These results may be a potential contribution for the development of the reinforced concrete project and its structural analysis.
\end{abstract}

Keywords: axial compressive strength; transition zones; anisotropy; support restriction; concrete. 


\section{INTRODUÇÃO}

As propriedades apresentadas pelo concreto têm origem em sua microestrutura interna, a qual expressa grande complexidade e heterogeneidade. Assim, o conhecimento da microestrutura do concreto se torna essencial para obter o melhor desempenho de suas propriedades, como resistência e durabilidade 0. Desse modo, é importante destacar que há microrregiões consideradas como o elo fraco do conjunto, responsáveis pelo início da proliferação de fissuras durante a solicitação de um elemento. Estas microrregiões que se localizam nas interfaces entre a pasta e o agregado são conhecidas como zonas de transição e podem ser tratadas como uma fase isolada da microestrutura do concreto, conforme MEHTA e MONTEIRO 0, MONTEIRO 0 e SHARMA E BISHNOI 0.

As zonas de transição são oriundas de um efeito chamado exsudação interna, o qual, de acordo NEVILLE 0, trata-se do processo de cura do material, visto que a água da mistura não é totalmente retida pelos outros constituintes do concreto e por ter menor densidade, tende a migrar até a superfície do elemento. Devido a este efeito, filmes de água se formam abaixo das partículas de agregado graúdo, acarretando em um aumento do fator água/cimento nestas regiões, tornando-as mais porosas e, portanto mais frágeis que o restante do conjunto 070. Conforme ANGELIN, LINTZ e BARBOSA 0 e ROSSIGNOLO 0 as zonas de transição possuem forte influência em propriedades fundamentais do concreto, como resistência mecânica e módulo de elasticidade, interferindo diretamente no modo de propagação de fissuras e permeabilidade do material, fazendo-se necessário cada vez mais a busca pela redução destas regiões frágeis.

Segundo VAN MIER 0, HUGHES e ASH 0 as zonas frágeis formadas sob as maiores partículas de agregados originam planos de clivagem suscetíveis à formação de fissuras. Quando o carregamento é aplicado paralelo à direção de lançamento do concreto, estas microrregiões localizadas nas zonas de transição se encontram em uma direção menos favorável para a propagação das microfissuras, pois estão abaixo dos agregados. Ao aplicar o carregamento perpendicular à direção de lançamento do concreto, os micros defeitos estão orientados na mesma direção em que o carregamento é aplicado, favorecendo uma rápida propagação de microfissuras, levando o corpo de prova à ruptura com pouca deformação e consequentemente com cargas reduzidas. Na Figura 1 é ilustrado como ocorre a propagação das fissuras à medida que se altera a direção do carregamento em relação ao lançamento do concreto nas formas.

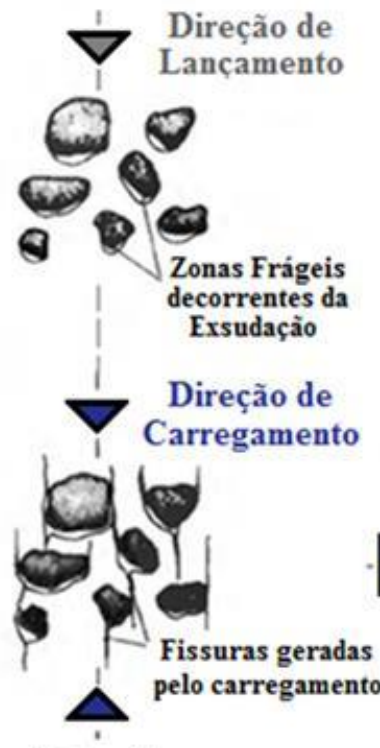

(a) Paralelo

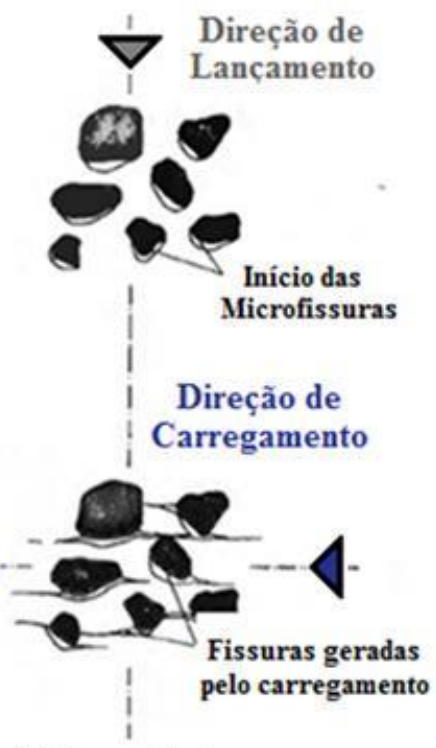

(b) Perpendicular

Figura 1: Propagação de fissuras quando o carregamento é aplicado (a) paralelamente e (b) perpendicularmente ao lançamento do concreto 0 .

Trabalhos como os de VAN MIER 0, HUGHES e ASH 0, LESHCHINSKY 0, HANSEN, LAURIDSEN e HOANG 0, YI, YANG e CHOI 0, e KOSTESKI et al. 0 analisaram a resistência de corpos de prova variando a direção de carregamento da amostra para diferentes classes de concretos. Os autores observaram que há um efeito anisotrópico nos ensaios, sendo que YI, YANG e CHOI 0 verificaram que elevar a resistência à compressão do concreto o efeito é reduzido. Em contrapartida KOSTESKI et al. 0 observaram, 
ao estudar concretos de baixa e média resistência, uma maior anisotropia nos concretos de média resistência. Ainda, os autores afirmam que um elemento de concreto pode possuir uma variação na resistência à compressão, que depende da direção a qual irá receber o carregamento, e então pode-se dizer que o material concreto apresenta anisotropia. Sendo assim estruturas moldadas em uma direção e solicitadas em outra podem ter sua resistência de cálculo comprometida 010-170.

Um exemplo de elementos com esta característica são pilares pré-moldados. Elementos como estes são convenientemente submetidos a ensaios para obtenção de parâmetros de cálculo e segurança, porém as amostras retiradas dos elementos para ensaios de resistência à compressão são obtidas perpendiculares à superfície da estrutura e devido à anisotropia do concreto, estas construções podem estar sendo analisadas equivocadamente, fato verificado por autores como HANSEN et al. 0 e ERGÜN e KÜRKLÜ 0.

Adicionalmente, as condições de contorno utilizadas nos testes de ensaios experimentais também podem modificar as conclusões obtidas sobre os valores corretos da resistência à compressão, visto que o atrito gerado na interface entre o corpo de prova e os pratos da máquina de ensaio resulta em uma modificação no modo de falha e nas propriedades mecânicas mensuradas dos corpos de prova, como explicado em VAN GEEL 0. Ao aumentar o atrito na interface, o deslocamento entre o elemento e máquina torna-se restrito em virtude das tensões de fricção nas extremidades da amostra, gerando um confinamento de tensões que forçam a um modo de falha padrão, com formato tronco-cônico 018-210. Na Figura 2, pode-se verificar como ocorre a distribuição de tensões e os modos de ruptura em corpos de prova submetidos à compressão axial em duas condições de contorno, mostrado inicialmente por BEZERRA et al. 0.

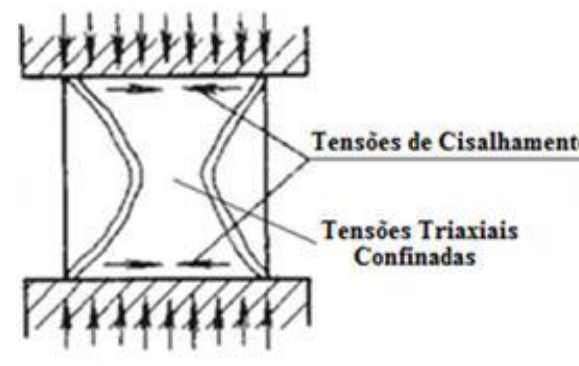

(a) Atrito máximo

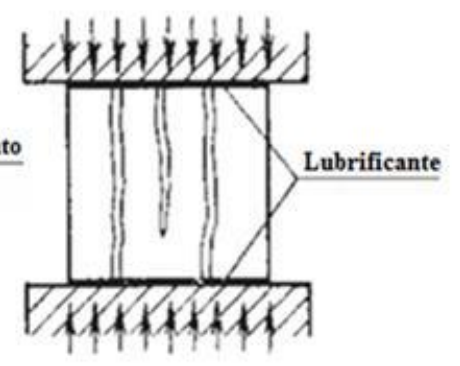

(b) Atrito minimo

Figura 2: Modo de falha das amostras de acordo com as condições de contorno: a) ensaio com fricção máxima e b) ensaio com fricção mínima 0 .

Autores como VAN MIER et al. 0, CARPINTERI, CIOLA e PUGNO 0, ZISOPOULOS, KOTSOVOS e PAVLOVIC 0 e SHAH, CHOI e JANSEN 0 estudaram o efeito da restrição nas extremidades de corpos de prova cilíndricos e prismáticos em contato com os pratos da máquina de ensaio, verificando a relação do comportamento mecânico destes com esbeltez e as condições de contorno impostas na execução do ensaio. Constataram que quando os ensaios são realizados com lubrificante na interface não há variação na resistência à compressão, assim como se verificou um decréscimo significativo de resistência quando comparado a ensaios em que existe fricção, além de que observaram uma notória redução de ductilidade da amostra quando minimizada a fricção nos ensaios. Entre outros, XU e CAI 0, INDELICATO e PAGGI 0 utilizaram modelos numéricos não lineares baseados no método dos elementos finitos para estudar o efeito do atrito na extremidade em amostras submetidas à compressão, obtendo resultados similares aos encontrados experimentalmente. Visto isso, constata-se que ao elevar o atrito nas faces de contato da amostra as mesmas apresentam uma resistência mais elevada devido à fricção proporcionar tensões de cisalhamento que atuam na interface entre os pratos da máquina de ensaios e o corpo de prova, gerando uma situação de confinamento no interior do elemento, o que consequentemente eleva a carga máxima dos mesmos 0.

Portanto, o presente estudo visa avaliar a anisotropia do concreto com base nas referências citadas, utilizando corpos de prova cúbicos, obtidos através de três traços de concreto, também avaliar a interferência das condições de contorno impostas no ensaio de compressão axial, visto que o atrito entre espécime e máquina de ensaio possui grande influência na resistência final do concreto. Em KOSTESKI et al. [15] foram utilizados corpos de prova cúbicos para estudar o efeito da direção do lançamento do concreto na resistência a compressão axial, da mesma forma que vários outros trabalhos anteriores. Já em KOSTESKI, MARANGON e RIERA [27] foi estudado o mesmo efeito utilizando corpos de prova cilíndricos. Os resultados encontrados nesses dois trabalhos são equivalentes, porém o segundo apresenta uma maior dificulda- 
de/complexidade de execução dos ensaios experimentais, além do elevado custo. Por este motivo, no presente trabalho, foram utilizados corpos de prova cúbicos.

\section{MATERIAIS E MÉTODOS}

Foram moldados corpos de prova a partir de três traços, projetados para resistência à compressão caraterística de 30MPa, 50MPa e 65MPa, denominados ao decorrer do trabalho de C30, C50 e C65, respectivamente. Os materiais utilizados para os três traços de concreto foram: agregado graúdo: brita 1 de origem basáltica extraída de uma jazida no município de Alegrete/RS, cujo diâmetro característico é $19 \mathrm{~mm}$ e módulo de finura de 6,77; agregados miúdo: utilizaram-se duas areias (Areia 1 e Areia 2) obtidas do leito do rio Ibicuí do Município de Manoel Viana/RS, sendo seus diâmetros característicos e módulos de finura 0,425 mm e 1,16 para Areia 1 e 0,6 mm e 1,38 para Areia 2, respectivamente. O cimento Portland utilizado foi do tipo CP V ARI. As composições dos traços de concretos são apresentados na Tabela 1. As relações a/c, conforme Tabela 1, foram necessárias para obter as resistências à compressão projetadas. A medida do abatimento do concreto C30 foi $10 \pm 2 \mathrm{~cm}$. Para manter a mesma trabalhabilidade, uma vez que diminuíram-se as relações água/cimento (A/C) para os traços C50 e C65, adicionou-se aditivo químico superplastificante. A análise das propriedades dos materiais citados anteriormente, moldagem e cura do concreto, foram realizadas de acordo com os procedimentos normativos da Associação Brasileira de Normas Técnicas (ABNT).

Tabela 1: Composições dos traços de concretos.

\begin{tabular}{ccccccc}
\hline TRAÇO & $\begin{array}{c}\text { CIMENTO } \\
\left(\mathrm{KG} / \mathbf{M}^{3}\right)\end{array}$ & $\begin{array}{c}\text { AREIA 1 } \\
\left(\mathbf{K G} / \mathbf{M}^{3}\right)\end{array}$ & $\begin{array}{c}\text { AREIA 2 } \\
\left(\mathbf{K G} / \mathbf{M}^{3}\right)\end{array}$ & $\begin{array}{c}\text { BRITA } \\
\left(\mathbf{K G} / \mathbf{M}^{3}\right)\end{array}$ & $\begin{array}{c}\text { SUPERPLASTIFICANTE } \\
(\% \text { DA MASSA DE CIMEN- } \\
\text { TO) }\end{array}$ & A/C \\
\hline C30 & 309,12 & 794,44 & - & 1078,83 & - & 0,64 \\
C50 & 445,59 & 338,65 & 414,40 & 1029,31 & $0,24 \%$ & 0,44 \\
C65 & 512,63 & - & 656,17 & 1132,91 & $0,80 \%$ & 0,34 \\
\hline
\end{tabular}

Na Figura 3 a) e b), pode-se observar os moldes prismáticos vazios e cheios de concreto utilizados para o presente estudo. Após um dia de idade, os corpos de prova foram desmoldados e levados à câmara úmida. Após 14 dias de cura em câmara úmida, cortaram-se os prismas $(10 \times 10 x 40 \mathrm{~cm}$ mostrados da Figura $3 \mathrm{c})$ com uma máquina de corte apropriada com serra diamantada, como mostra a Figura 3 d). Para a regularização das superfícies dos cubos (de 10x10x10 cm, Figura 3 e) realizou-se o capeamento com uma fina camada de argamassa. Na Figura 3 f) pode-se observar a execução do capeamento dos corpos de prova. 

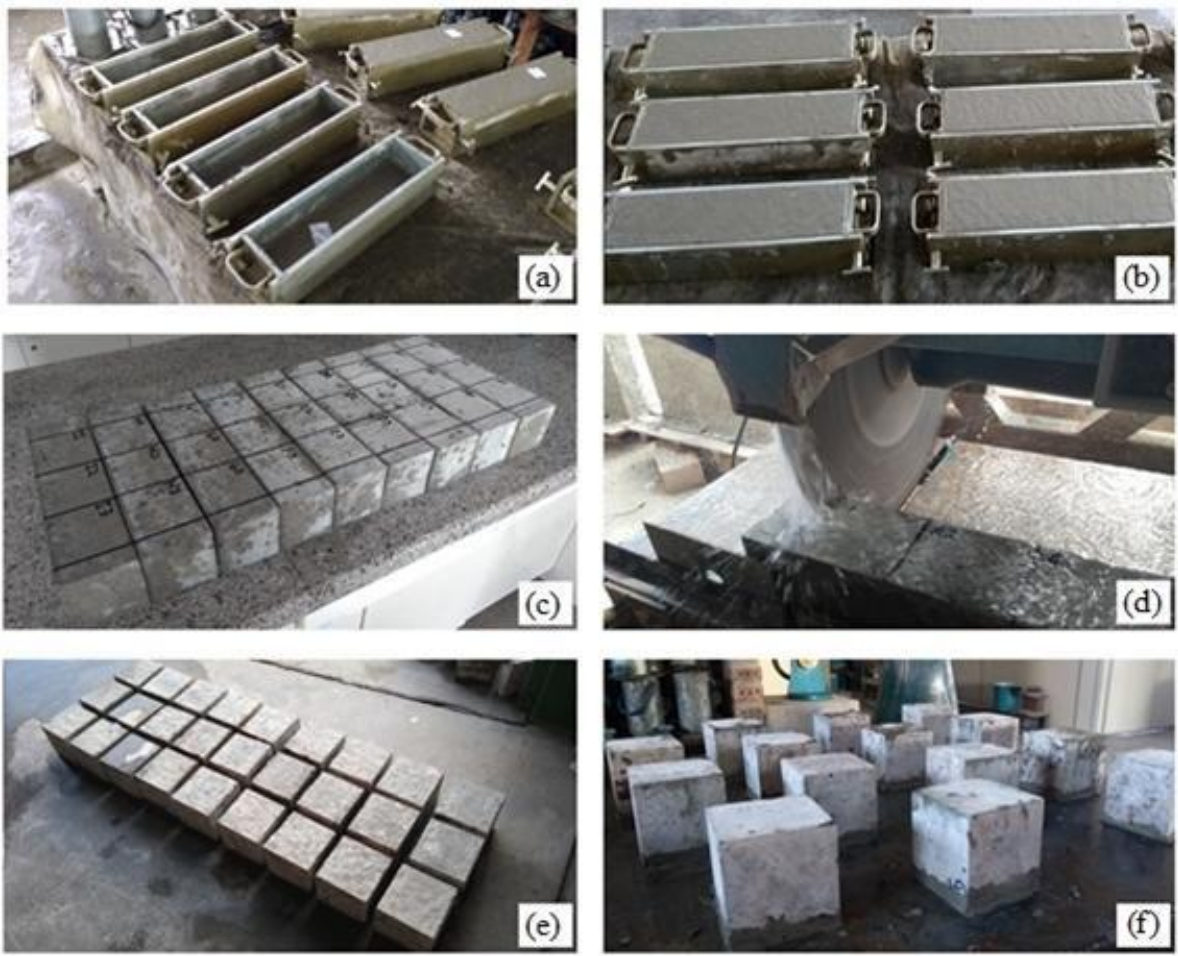

Figura 3: Passo a passo da moldagem: a) moldes prismáticos utilizados, b) moldes após o lançamento e adensamento do concreto, c) desforma de alguns prismas, d) máquina de corte, e) corpos de prova cúbicos e f) corpos de prova capeados.

Foram moldados 5 corpos de prova cilíndricos $(10 \times 20 \mathrm{~cm})$ para cada traço, com o intuito de realizar o ensaio de resistência à compressão axial padrão. Os ensaios de compressão axial nos cilindros foram executados conforme rege a NBR 57390 usando uma máquina de ensaios universais da marca INSTRON, SATEC séries, modelo 5590-HVL, com capacidade máxima de $1500 \mathrm{kN}$.

Para todos os traços dos concretos estudados, foram ensaiados à compressão axial nas direções paralelo (Caso P) e perpendicular ou normal (Caso N) ao lançamento do concreto. Para os concretos C30 e C65 ensaiaram-se amostras em duas condições de contorno, uma com a utilização de graxa nas interfaces (Caso G), outra com placas de aço coladas de espessura de $3 \mathrm{~mm}$ (Caso C) e também na forma tradicional, sem nenhum material entre o concreto e os pratos da máquina (Caso T). Além disso, para o traço C65, analisou-se a resistência à compressão em relação a direção de lançamento do concreto, correlacionando os Casos: P, N, G e C. Para cada um dos casos citados foram moldados e ensaiados 10 corpos de prova.

As amostras ensaiadas pelo Caso $\mathrm{P}$ foram colocadas na posição de ensaio na mesma disposição em que foram concretados e postos a cura, como mostra a Figura 4 a); para as amostras ensaiadas através do Caso N, rotacionaram-se $90^{\circ}$ da posição em que foram concretados (Figura 4 b). Na Figura 4, observa-se a modificação da orientação das zonas de transição (zonas frágeis) em relação à aplicação de carga, a qual influencia na resistência à compressão do concreto, uma vez que essas se encontram orientadas em uma disposição normal ao lançamento do concreto sob os agregados graúdos. 


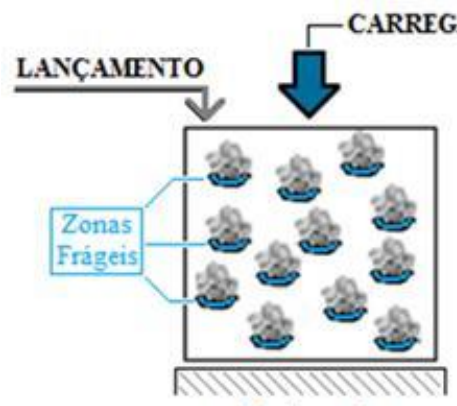

(a) Caso P

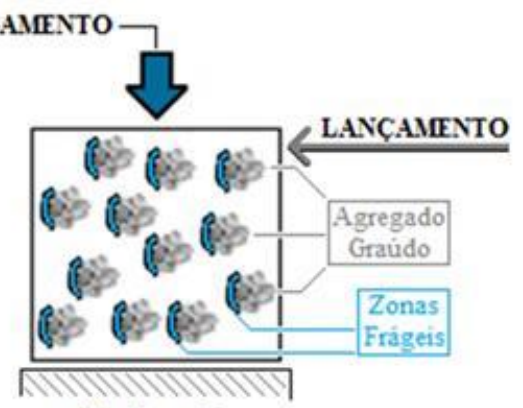

(b) Caso N

Figura 4: a) aplicação do carregamento paralelo ao lançamento de concreto (Caso P) e b) aplicação do carregamento perpendicular ao lançamento (Caso $\mathrm{N})$.

Os ensaios de compressão axial executados com as diferentes condições de contorno, sendo o Caso C representando atrito máximo entre os elementos de concreto e os pratos da máquina de ensaios e o Caso G o ensaio caracterizando o atrito mínimo, podem ser visualizados na Figura 5.

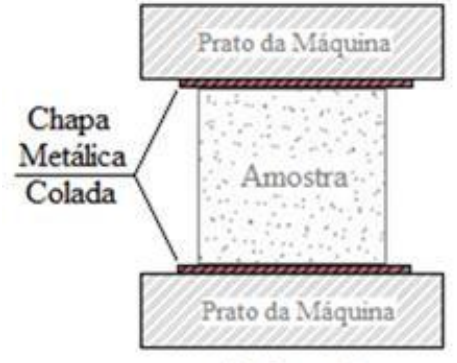

(a) Caso C

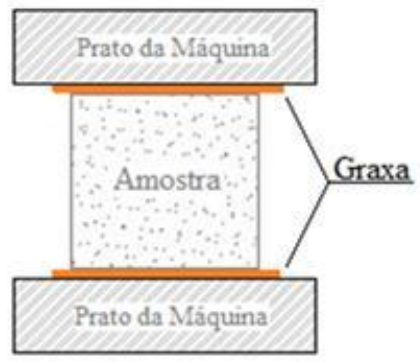

(b) Caso G

Figura 5: a) condição de atrito máximo (Caso C); b) condição de atrito mínimo (Caso G).

Para todos os resultados dos ensaios de compressão axial foram realizadas análises estatísticas das variâncias (ANOVA) e a comparação múltipla de médias pelo método de Tukey, com um nível de confiança de 95\%. Através dessas análises, é possível concluir se ocorreu alguma variância significativa entre os resultados encontrados.

\section{RESULTADOS E DISCUSSÕES}

\subsection{Resistência à compressão axial: corpos de prova cilíndricos padrão}

$\mathrm{Na}$ Tabela 2, pode-se observar os resultados encontrados para os ensaios experimentais dos corpos de prova cilíndricos padrão, para os três traços utilizados nesse trabalho. Na Figura 6, são apresentadas as curvas de tensão de compressão axial versus deformação. Pode-se observar que os valores de resistência à compressão axial obtidos nos ensaios são próximos aos valores projetados (30, 50 e 65MPa). A partir destas, é possível verificar que o módulo de elasticidade dos concretos C30 e C50 apresentam proximidade, já o traço C65 demonstra uma rigidez consideravelmente superior. As características de cada concreto, verificadas pelas curvas apresentadas na Figura 5, servirão de base para análise dos resultados obtidos pelos corpos de prova cúbicos.

Tabela 2: Resistências à compressão axial, módulos de elasticidade e seus respectivos coeficientes de variação, dos traços estudados quando ensaiados em amostras cilíndricas.

\begin{tabular}{ccccc}
\hline TRAÇO & $\begin{array}{c}\text { TENSÃO MÉDIA } \\
\text { (MPA) }\end{array}$ & CV (\%) & $\begin{array}{c}\text { MÓDULO DE ELAS- } \\
\text { TICIDADE (GPA) }\end{array}$ & CV (\%) \\
\hline C30 & 29,42 & 2,09 & 33,45 & 1,68 \\
C50 & 47,67 & 0,55 & 33,65 & 7,32
\end{tabular}




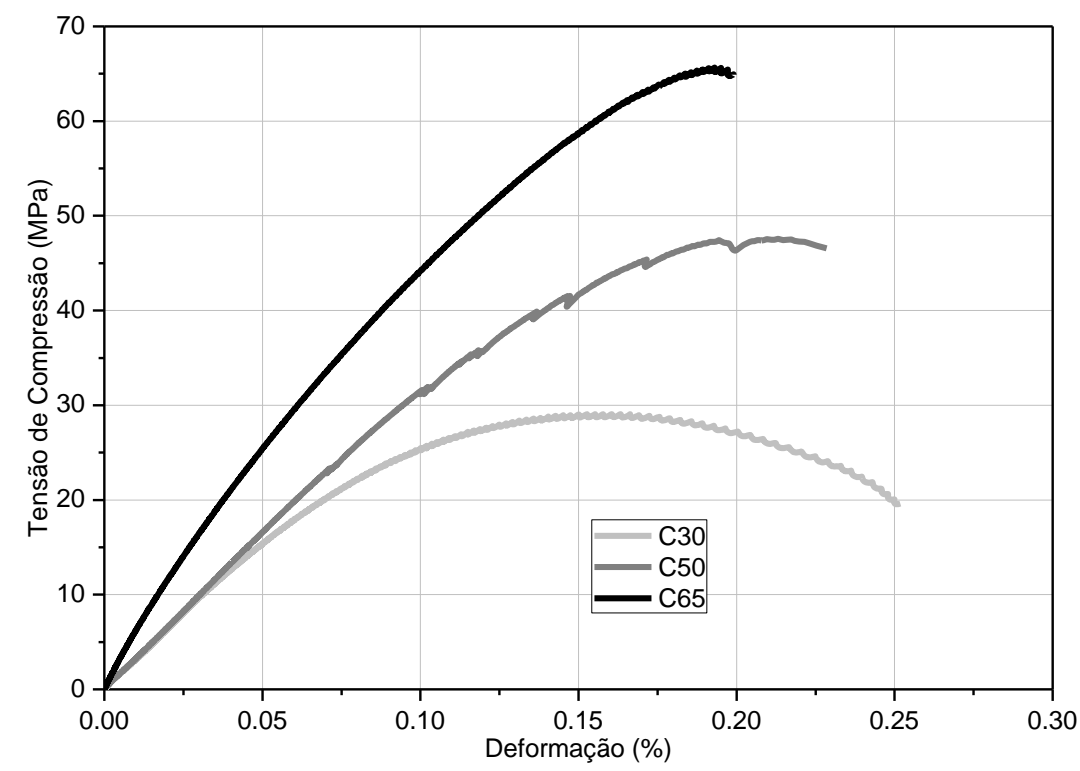

Figura 6: Curvas de tensão de compressão versus deformação dos corpos de prova cilíndricos para os três tipos de traço.

\subsection{Resistência à compressão axial: corpos de prova cúbicos}

Na Figura 7, estão apresentados os resultados encontrados do ensaio de compressão axial, para os corpos de prova cúbicos e a média da resistência dos corpos de prova cilíndricos, para os três traços analisados levando em consideração a direção de carregamento (Casos P e N).

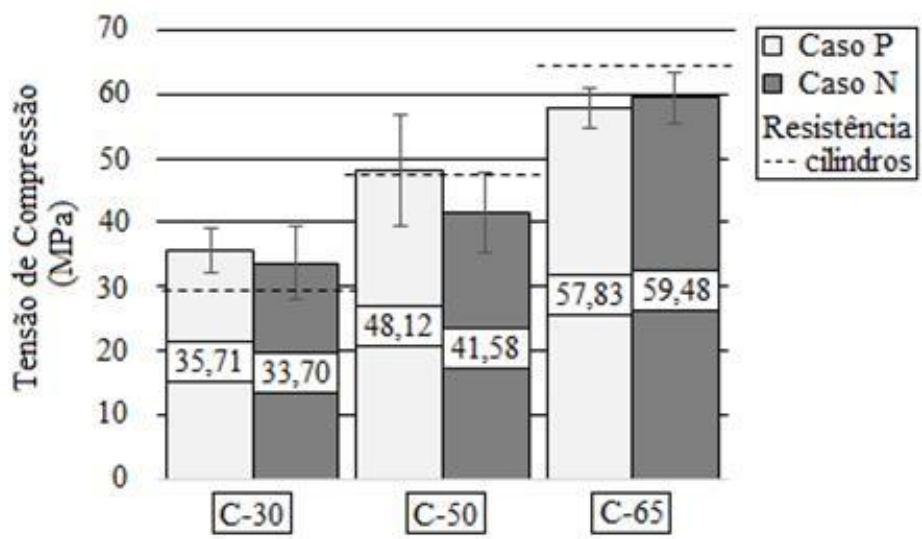

Figura 7: Resistência dos corpos de prova de acordo com a direção de carregamento em relação ao lançamento.

Verificou-se que para o traço $\mathrm{C} 30$, ocorreu um aumento de $6 \%$ na resistência à compressão axial no Caso P (quando o carregamento é aplicado paralelo à direção de lançamento). Cabe salientar que esta diferença não é significativa quando utilizado o método de Tukey. A baixa resistência do concreto pode ser atribuída a uma maior heterogeneidade da mistura, ou seja, a argamassa apresenta pouca resistência em relação aos agregados, portanto, a ruptura deste concreto não necessariamente ocorre nas zonas de transição.

O traço C50 é 16\% mais resistente quando o ensaio é realizado pelo Caso P. Esta diferença é significativa quando realizado a análise de variâncias. O traço C50 tem resistência mais elevada e, em seu processo de ruptura, o elemento tende a fissurar nos pontos críticos, isto é, nas zonas de transição. Isso ocorre devido à argamassa e ao agregado apresentarem resistências elevadas, a propagação das fissuras tendem a se concentrar nas zonas de transição. Esse resultado confere com o que foi encontrado por KOSTESKI et al. 0. 
Para o traço C65, a resistência à compressão média dos corpos de prova ensaiados foi 3\% maior quando comparado com os resultados obtidos pelos ensaios realizados pelo Caso N (Carregamento perpendicular ao lançamento). Novamente, não é possível observar diferença significativa entre os dois casos. Este concreto de alta resistência possui um fator a/c muito baixo. Como as zonas de transição são fruto da exsudação interna do concreto e, por consequência disso, quanto menor a quantidade de água utilizada para a produção do mesmo, menor será a exsudação ocorrida e, consequentemente, menor a concentração da água sob os agregados, ou seja, assim o defeito de aderência será menor entre agregado e pasta.

Sendo assim, verificou-se uma maior relevância quanto a anisotropia para o concreto C50, o que não quer dizer que as zonas de transição não existam ou não influenciem nos ensaios dos concretos C30 e C65, mas sim que estes são menos afetados, devido aos fatores citados, ao ponto das zonas frágeis não se tornarem determinantes para o rompimento dos corpos de prova ensaiados.

\subsection{Resistência à compressão axial: variação das condições de contorno}

Na Figura 8, estão apresentados os resultados encontrados com as três condições de contorno utilizadas, Casos G, C e T. Esses resultados foram obtidos para os concretos C30 e C65.

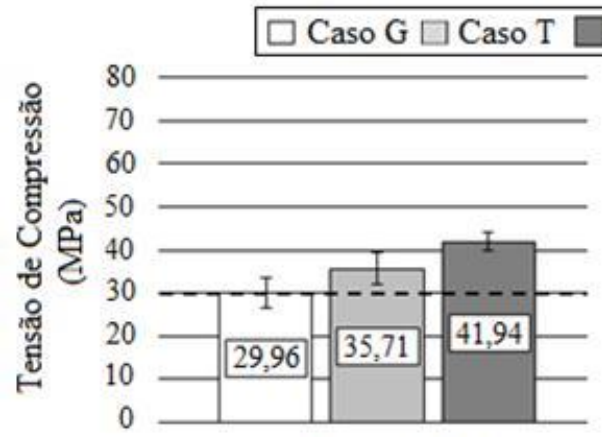

(a) Traço $\mathrm{C} 30$

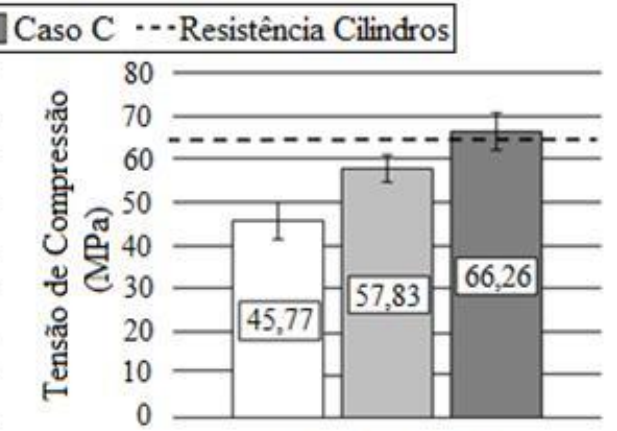

(b) Traço $\mathrm{C} 65$

Figura 8: Resultados obtidos dos ensaios de compressão axial nas diferentes condições de contorno a) traço C30 e b) traço C65.

Realizando uma análise de variância (Anova) com todos os resultados mostrados na Figura 8, entre os traços C30 e C65 e as condições de contorno dos Caso G, T e C, verifica-se diferenças significativas entre todos os fatores analisados. Conforme mostra a Figura 8, pode ser observada uma diferença da ordem de $40 \%$ entre os casos $\mathrm{C}$ e G, independente do tipo da classe de concreto utilizado. É interessante observar que o caso $\mathrm{T}$ no traço $\mathrm{C} 30$ tem um comportamento médio entre os casos $\mathrm{G}$ e $\mathrm{C}$, isto é um atrito intermediário. No entanto, o caso $\mathrm{T}$, para o traço $\mathrm{C} 65$, encontra-se a um terço do caso $\mathrm{C}$ e dois terços do caso $\mathrm{G}$, mais próximo do atrito máximo que do mínimo.

A Figura 9, mostra as imagens que caracterizam os modos de falha dos corpos de prova para cada concreto nos casos G, T e C. Os modos de ruptura encontrados estão coerentes com os apresentados por BEZERRA et al. 0 (Figura 2). O modo de ruptura dos corpos de prova ensaiados com aplicação de graxa nas faces apresentou inúmeras fissuras verticais e grande deformação dos elementos. Os corpos de prova, restringidos por placas e cola, apresentaram a fratura padrão, em formato de ampulheta, devido ao confinamento de tensões triaxiais, conforme explicaram CARPINTERI, CIOLA e PUGNO 0. 


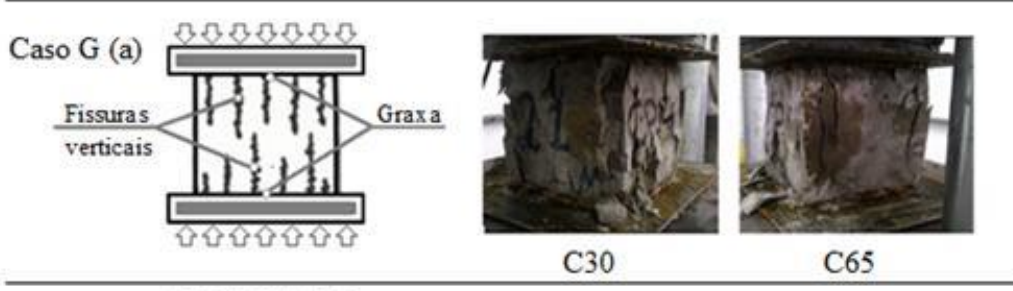

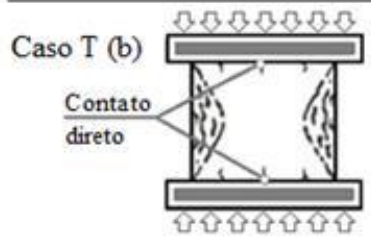

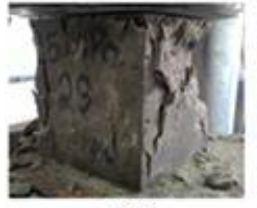

$\mathrm{C} 30$

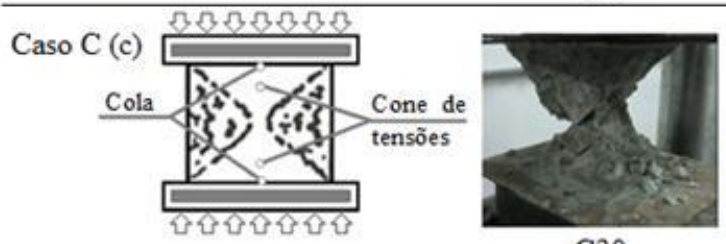

$\mathrm{C} 30$

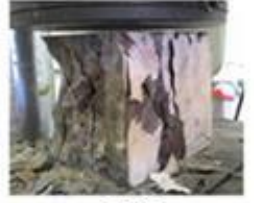

$\mathrm{C} 65$

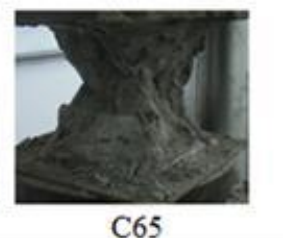

Figura 9: Modo de fratura das amostras em diferentes condições de contorno: a) Ensaio com atrito mínimo (Caso G), b) Ensaio de compressão tradicional (Caso T) e c) Ensaio com atrito máximo (Caso C).

Os resultados apresentados pelos concretos C30 e C65, assim como as imagens dos modos de ruptura, demonstram coerência de acordo com a bibliografia [190. Esta constatação chama a atenção aos ensaios de resistência à compressão padrão (linha de traços na Figura 8), que é o valor da resistência do concreto que deve ser compatível com a resistência definida em projetos de dimensionamento de estruturas. Como verificado nos resultados deste tópico, de acordo com o atrito imposto ao ensaio de compressão, a resistência encontrada pode variar.

Assim, esses resultados são de grande relevância para questões práticas da engenharia, como também para o embasamento teórico do outro tema abordado neste trabalho, que é a anisotropia. Verificou-se que os corpos de prova cúbicos sofrem um confinamento à medida que se eleva à fricção, isto revela que, para ensaios do Caso $\mathrm{C}$, é provável que não ocorra o efeito, pois o esforço interno não vai ocorrer em uma única direção, ou seja, não se trata de um esforço axial. Portanto, a resistência das amostras cúbicas independem da disposição das zonas de transição em relação a direção de carga, visto que o esforço interno do material está ocorrendo em diferentes direções.

\subsection{Interação entre a direção de carregamento e as condições de contorno}

Para o traço C65, executaram-se ensaios variando a direção do carregamento pelos Casos $\mathrm{P}$ e $\mathrm{N}$ e mudando as condições de contorno através dos Casos G e C para, assim, verificar se ocorre alguma variação de resistência entre os mesmos. Na Figura 10, são apresentados os resultados encontrados.

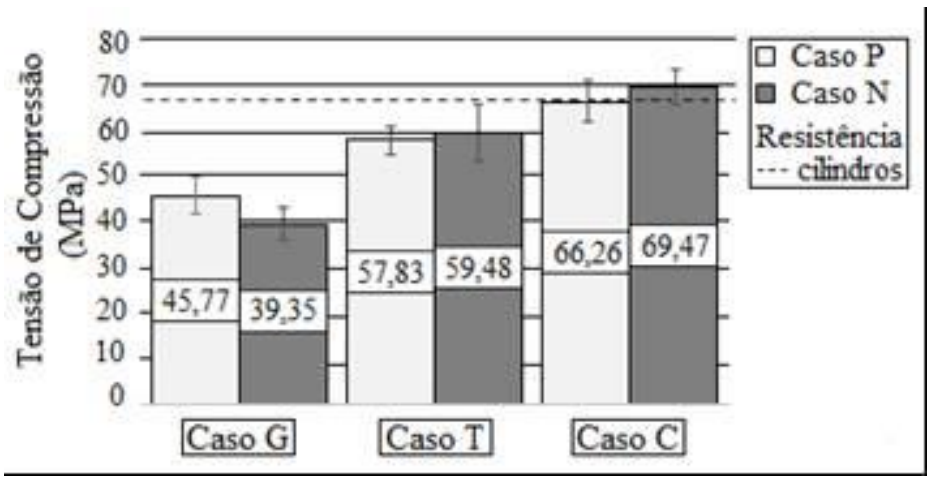

Figura 10: Resistência a compressão axial de acordo com a direção de carregamento variando as condições de contorno para o concreto C65. 
Pode-se perceber, através da Figura 10, uma modificação no efeito da direção do carregamento com a mudança das condições de contorno, porém não significativa. Assim, quando se tem o mínimo atrito, a resistência à compressão axial obtida no caso $\mathrm{P}$ é maior que o $\mathrm{N}(16 \%)$, enquanto que, quando se tem o atrito máximo, este comportamento se inverte ou deixa de ter influência.

No caso C, devido ao confinamento de tensões ocasionadas pela fricção, são originadas tensões triaxiais, as quais representam um estado de tensões composto por forças que atuam em três eixos distintos. Em razão disso, independente da direção de aplicação do carregamento nas amostras, as forças atuantes estarão agindo em várias direções. Sendo assim, as disposições das zonas de transição em relação à direção do carregamento acabam por não ter influência na resistência das amostras. Este resultado é muito semelhante ao encontrado no caso $\mathrm{T}$, onde também não se verificou uma diferença significativa da variação da direção do carregamento. A Figura 11 apresenta o processo de fissuração durante o ensaio de compressão axial para concreto C65 nos casos P e N nas condições de atrito máximo (Caso C) e mínimo (Caso G).

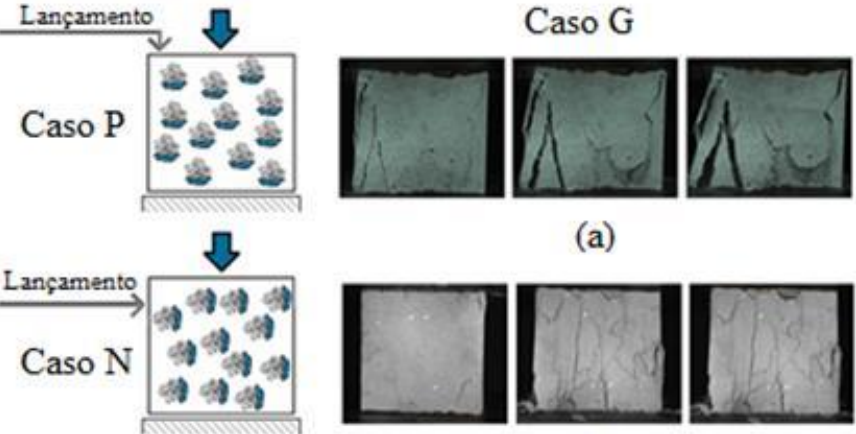

(c)

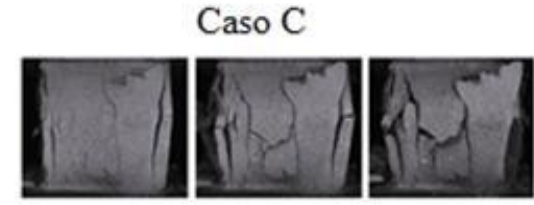

(b)

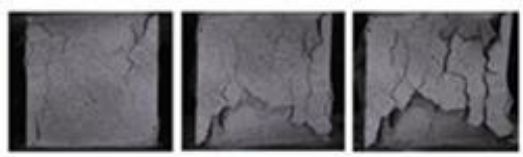

(d)

Figura 11: Modelo de fissuras: a) paralelo ao lançamento do concreto sem restrição, b) perpendicular ao lançamento do concreto sem restrição, c) paralelo ao lançamento do concreto com restrição e d) perpendicular ao lançamento do concreto com restrição.

Dentre as constatações observadas, pode-se concluir que, à medida que se reduz o atrito na interface entre corpo de prova e máquina de ensaio, o efeito de anisotropia se torna mais aparente devido à predominância de esforço unidirecional no elemento, agindo paralelo ou perpendicular à disposição das zonas de transição de acordo com a direção de carga. Assim, verifica-se a redução da resistência do concreto gradualmente com o alívio da fricção.

\section{CONCLUSÕES}

Com a realização deste trabalho, pode-se verificar a variação de resistência mecânica dos corpos de prova de acordo com a direção do carregamento e conclui-se que, tanto para concretos de baixa resistência como para de alta resistência, esta variação não é relevante devido à mínima exsudação interna dos concretos mais resistentes e a expressiva heterogeneidade dos concretos pouco resistentes. Para concretos de média resistência, no caso do estudo o concreto $\mathrm{C} 50$, a variação apresentou-se relevante, perdendo resistência à medida que o carregamento é aplicado (perpendicular ao lançamento do concreto). Isso indica que além da relação água/cimento nos traços de concreto, existem outros aspectos a serem avaliados para quantificar a influência da divergência da aplicação de carga em relação à de lançamento na resistência de elementos estruturais de concreto.

Quanto aos ensaios com duas condições de contorno, verificou-se que à medida que se aumentava o atrito em um ensaio de compressão axial, maior é a resistência do elemento. No caso de impor o mínimo de atrito ao ensaio, a resistência das amostras reduz consideravelmente, sendo que desta ocorre o ensaio de compressão axial propriamente dito, eliminando ou reduzindo a probabilidade de um confinamento de tensões triaxiais.

Para os ensaios onde foi analisada a variação de resistência mecânica de corpos de prova de acordo com a direção de carregamento em duas condições de contorno, constatou-se que quando os ensaios são executados sem restrição, há uma variação significativa de resistência à medida que se muda a direção de carregamento em relação ao de lançamento (moldagem). Este fato não foi verificado para ensaios com restrição. Isso leva a constatação de que à medida que se eleva o atrito nas interfaces da amostra, a probabilidade do 
concreto apresentar anisotropia de acordo com a direção do carregamento é mínima.

Portanto, com base nos resultados encontrados é possível afirmar a contribuição do estudo em termos de análise estrutural e desenvolvimento de projetos de concreto armado, pois, mesmo sendo o concreto um material amplamente caracterizado por seu comportamento isotrópico, verificou-se neste trabalho que devido a efeitos gravitacionais, os quais naturalmente ocorrem no lançamento do concreto, o mesmo pode apresentar comportamento anisotrópico ao longo de seu endurecimento e, devido a isto, pode variar sua resistência à compressão de acordo com o ângulo de aplicação do carregamento em relação ao de lançamento. Através dos ensaios, variando as condições de contorno, verificou-se uma notória discrepância entre as resistências à compressão das amostras, fato que ressalta a importância da padronização e redução do atrito em ensaios de compressão normatizados, garantindo assim que o corpo de prova esteja submetido somente à carga uniaxial, propiciando melhor compatibilidade com a resistência característica calculada para projetos de engenharia.

Por fim, deve-se ressaltar que este trabalho além de ratificar e enriquecer o que já foi determinado por outros autores quanto à anisotropia, proporciona uma análise do efeito para diferentes resistências de concreto e ainda correlaciona estas às condições de contorno impostas durante a execução dos ensaios de compressão axial.

\section{AGRADECIMENTOS}

Os autores agradecem às empresas Pedro Rosada e Rosário Postes pela doação da brita e o cimento utilizados para realizar esta pesquisa. A colaboração do Engenheiro Civil Francisco Keche dos Santos foi muito importante para a realização deste trabalho. Também agradecem o apoio da UNIPAMPA, CNPq, CAPES e FAPERGS. O presente trabalho foi realizado com apoio da Coordenação de Aperfeiçoamento de Pessoal de Nível Superior - Brasil (CAPES) - Código de Financiamento 001.

\section{BIBLIOGRAFIA}

[1] MEHTA, P. K., MONTEIRO, P. J. M., "Concreto: Microestrutura, Propriedades e Materiais", In: $3^{\text {a }}$ ed. Instituto Brasileiro de Concreto - IBRACON, São Paulo - SP, 2008.

[2] MONTEIRO, P. J. M., "Microstructure of concrete and its influence on the mechanical properties", Tese de D.Sc., University of California, Berkeley, 1985.

[3] SHARMA, M., BISHNOI, S., "The Interfacial Transition Zone: Microstructure, Properties, and Its Modification”. In: Rao A., Ramanjaneyulu K. (eds) Recent Advances in Structural Engineering, v. 2. Lecture Notes in Civil Engineering, Springer, Singapore. 2019.

[4] NEVILLE, A. M., Propriedades do concreto, 5a ed. São Paulo, 2016.

[5] LIJUAN, K., YUANBO, D., "Interfacial Interaction of Aggregate-Cement Paste in Concrete", Journal of Wuhan University of Technology-Mater. Sci. Ed, v. 30, n.1, pp. 117-121, 2015.

[6] ELSHARIEF, A., COHEN, M. D., OLEK A., "Influence of aggregate size, water cement ratio and age on the microstructure of the interfacial transition zone", Cement and Concrete Research, v. 33, n.11, pp. 18371849, 2003.

[7] REDA, M. M., SHRIVE, N. G., GILLOTT, J. E., "Microstructural investigation of innovative UHPC", Cement and Concrete Research, v. 29, n.3, pp. 323-239, 1999.

[8] ANGELIN, A. F., LINTZ, R. C. C., BARBOSA, L. A. G., "Use of expanded clay and silica fume in improvement of mechanical, physical and thermal performances of structural lightweight concretes", Revista Matéria, Rio de Janeiro - RJ, v. 22, 2017.

[9] ROSSIGNOLO, J. A. "Avaliação da zona de transição interfacial pasta-agregado leve em concretos com sílica ativa e látex SBR”, Revista Matéria, Rio de Janeiro - RJ, v. 12, n. 3, pp. 532-540, 2007.

[10] VAN MIER, J. G. M., Strain-softening of concrete under multiaxial loading conditions, Eindhoven: Technische Hogeschool Eindhoven, 1984.

[11] HUGHES, B. P., ASH, J. E., "Anisotropy and failure criteria for concrete", Matériaux et Constructions, v. 3, n.6, pp. 371-374, 1970.

[12] LESHCHINSKY, A. M. "Anisotropy of concrete strength". Cement, Concrete and Aggregates, v. 12, n. 2, pp. 117-119, 1990. 
[13] HANSEN, S.G., LAURIDSEN, J.T., HOANG, L.C., "Experimental and statistical investigation of the compressive strength anisotropy in structural concrete", Cement and Concrete Research, v. 107, pp. 304-316, 2018.

[14] YI, S.T., YANG, E.I., CHOI, J.C., "Effect of specimen sizes, specimen shapes, and placement directions on compressive strength of concrete", Nuclear Engineering and Design, v. 236, n.2, pp. 115 - 127, 2005.

[15] KOSTESKI, L. E., MARANGON, E., RIERA, J. D., et al., “Assessment of concrete anisotropy in relation to the direction of casting", Revista Sul-Americana de Engenharia Estrutural, v.15, n. 2, pp. 37-45, 2018.

[16] JOHNSTON, C. D. “Anisotropy of Concrete and Its Practical Implications”, Highway Research Record n. 423, pp. 11-16, 1973.

[17] ERGÜN, A., KÜRKLÜ, G., "Assessing the Relationship between the Compressive Strength of Concrete Cores and Molded Specimens”, Gazi University Journal of Science, v. 25, n.3, pp. 737-750, 2012.

[18] VAN GEEL, E., "Concrete Behaviour in Multiaxial Compression: Experiment al Research", Tese (doutorado) - Faculdade de Arquitetura da Universidade de Tecnologia de Eindhoven, Departamento de Design Estrutural, Eindhoven, pp. 178, 1998.

[19] VAN MIER J. G. M., SHAH, S. P., ARNAUD, M., et al., "Strain-softening of concrete in uniaxial compression”, Materials and Structures/Matériaux et Constructions, v. 30, pp. 195-209, 1997.

[20] MATTEI, N. J., MEHRABADI, M. M., ZHU, H. A., "Micromechanical constitutive model for the behavior of concrete", Mechanics of Materials, v. 39, n.4, pp. 357-379, 2007.

[21] BEZERRA, U. T., ALVES, S. M. S., BARBOSA, N. P., et al., "Corpo de prova na forma de ampulheta: resistência à compressão de concretos e argamassas, análise numérica e experimental", Ibracon structures and materials jornal, v. 9, pp. 510-524, 2016.

[22] CARPINTERI, A., CIOLA, F., PUGNO, N. "Boundary element method for the strain-softening response of quasi-brittle materials in compression”, Computers and Structures, v. 79, n.4, pp. 389-401, 2001.

[23] ZISOPOULOS, P. M., KOTSOVOS, M. D., PAVLOVIC, M. N. "Deformational behaviour of concrete specimens in uniaxial compression under different boundary conditions", Cement and concrete research, v. 30, n. 1, pp. 153-159, 2000.

[24] SHAH, S.P., CHOI, S., JANSEN, D.C. "Strain softening of concrete in compression. Proceedings of Fracture Mechanics of Concrete Structures", AEDIFICATIO Publishers, Freiburg, v. 3, pp. 1827-1841, 1996.

[25] XU, Y., CAI, M., "Numerical simulation of end constraint effect on post-peak behaviors of rocks in uniaxial compression", In: 49th US Rock Mechanics/Geomechanics Symposium. American Rock Mechanics Association, 2015.

[26] INDELICATO, F., PAGGI, M., "Specimen shape and the problem of contact in the assessment of concrete compressive strength", Materials and Structures, v. 41, n. 2, 2008.

[27] KOSTESKI, L.E., MARANGON, E., RIEIRA, J.D., “Assessment of concrete anisotropy by means of compression and indirect tensile tests", RIEM - IBRACON Structures and Materials Journal, [s.1.], v. 12, n. 5, apr. 2019. ISSN 1983-4195. Available at:

<http://www.revistas.ibracon.org.br/index.php/riem/article/view/1536>.

[28] ASSOCIAÇÃO BRASILEIRA DE NORMAS TÉCNICAS, NBR-5739 - Concreto - Ensaio de compressão de corpos-de-prova cilíndricos. Rio de Janeiro, 1994.

\section{ORCID}

Matthews Vargas Vaucher Bandeira https://orcid.org/0000-0001-8561-528X

Raquel Caroline Zydeck

https://orcid.org/0000-0003-3407-5783

Luis Eduardo Kosteski

https://orcid.org/0000-0002-5168-6909

Ederli Marangon

https://orcid.org/0000-0003-0812-8095 